\title{
Effect of Dimension \& Material Composition on Transmission Coefficient and Tunneling Current of Double Quantum Barrier Structure with Band Nonparabolicity
}

\author{
Arpan Deyasi and Gourab Kumar Ghosh
}

\begin{abstract}
Transmission coefficient, eigen states and tunneling current density of a potentially symmetric quantum double barrier structure has been numerically computed using transfer matrix technique for qualitative analysis of resonant tunneling probability when realistic band structure of higher band gap material is taken into account. GaAs/Al $G a_{1-x} A s$ material composition is taken as an example for calculation, and thickness of the barrier and well regions are varied along with material compositions of $A l_{x} G a_{1-x}$ As to study the effect on electrical parameters; and also to observe the existence of quasi-bound states. Effective mass mismatch at junctions is considered following envelope function approximation, and conduction band discontinuity is taken into account for computational purpose. Under low biasing condition, negative differential regions (NDR) can be obtained which speaks in favor of tunneling current.
\end{abstract}

Keywords--- Double Quantum Barrier, Transfer Matrix Method (TMM), Transmission Coefficient, Eigen Energy, Tunneling Current, Negative Differential Resistance (NDR)

\section{INTRODUCTION}

$\mathrm{T}$ HE present research activities in VLSI domain regarding shrinking of MOSFET dimension is deviated away from Moore's law, and new concepts of physics has been introduced to replace the existing VLSI-based electronics. By reducing the device size comparable to electron wavelength, spatial confinement along one, two or three dimensions can be achieved in semiconductor hetero structure devices. This may help to physically realize quantum well, wire or dot structures, which have numerous electronic and photonic applications [1$3]$. The region of confinement is coupled to the external world through tunneling barriers which vividly reflects the dominance of the quantum effects in the transport phenomena. Under suitable dimensional configuration and material composition, the discrete eigen states can exhibit resonant tunneling even at low biasing condition. The likelihood that

\footnotetext{
Arpan Deyasi, Assistant Professor, Department of Electronics \& Communication Engineering, RCC Institute of Information Technology, Kolkata,India,E-mail: deyasi_arpan@yahoo.co.in

Gourab Kumar Ghosh, P.G Student, Physics Department, Calcutta University, Kolkata, India, E-mail: gourabghosh_ph@gmail.com
}

the electron will pass through the barrier is given by the transmission coefficient, and is physically realizable in the device by measuring tunneling current. In quantum well-like structures, this physical insight already generated various novel microscopic applications [4-6].

Esaki and Tsu made the pioneering work by proposing semiconductor symmetric double barrier structure [7] where electronic transport proceeds via resonant tunneling mechanism. Later they suggested multiple quantum well structures, leading to superlattices [8], where a series of energy levels and associated subbands are formed due to the quantization of carriers in the direction of confinement. Computation for transmission coefficient carried out by Chanda [9], Christodoulides [10], and later Scandella [11] was without considering the effect of material parameters, which was later realized by Chang [12] Read [13]. They independently studied resonant tunneling probability in semiconductor double barrier structure for different material parameters. Wessel [14] calculated the same for thin barrier considering the $\mathrm{GaAs} / \mathrm{Al}_{\mathrm{x}} \mathrm{Ga}_{1-\mathrm{x}} \mathrm{As}$ material composition.

Band nonparabolicity is one of the most important feature should be taken into account for computation of electrical parameters of quantum well structures along with conduction band discontinuity and envelope function approximation [15], as it affects the eigen states, and hence, tunneling current. In 1-D confined structures, displacement of energy levels from band-edge should be considered for tailoring transport properties, and hence first-order non parabolic E- $\kappa$ relationship plays a vital role in determining transmission coefficient and current density for the device along with incorporation of physical parameters; and relative interdependency of these data should be taken into account for mathematical modeling. Miller [16] investigated the effect of conduction band non parabolicity on eigen energies using Kane's two-band model, Hiroshima [17] considered non parabolicity factor as a function of material parameters of the ultrathin layers. Nelson [18] considered energy-dependent effective mass approach to evaluate NP effect on transmission probabilities, and finite element method was introduced by Dave [19] for more precise estimation.

Computation of transmission coefficient and current density may be analyzed by solving time-independent Schrödinger's equation with proper boundary conditions. Existence of bound and quasi-bound states can be obtained 
from this analysis [20]. Double barrier resonant tunneling structure can be analyzed by several numerical techniques such as Variational Method [21], Airy's function approach [22-23], Finite Element Method (FEM) [24], Transfer Matrix Technique (TMT) [1], [4], [6], [22], [25]-[27], Weighted Potential Method (WPM) [28]. Comparing all these methods, TMT is considered as one of the effective and accurate method by eminent researchers. Resonant tunneling phenomenon for these devices under applied field [1], [5], [29]-[30] provides a theoretical estimation about transmission coefficient and tunneling current.

The present paper deals with transmission coefficient, eigen energies and tunneling current characteristics for potentially symmetric double quantum barrier structure. Well and barrier dimensions are independently varied to observe the effects on those electrical parameters, and material parameter of the barrier region is also varied to observe the same. Transfer matrix technique is adopted for numerical analysis. NP effect upto first order for barrier material is considered to increase the accuracy of the calculation, along with the consideration of conduction band discontinuity and effective mass mismatch. This helps to simulate the electrical parameters for close-to-realistic band diagram. Existence of quasi-bound states is verified along with the bound states. GaAs/ $/ \mathrm{Al}_{\mathrm{x}} \mathrm{Ga}_{1-\mathrm{x}} \mathrm{As}$ material composition is taken for the analysis. Peak and valley voltages are calculated from current profiles to estimate peak-to-valley ratio, and also to study the geometrical nature of NDR region. Rectangular potential profiles are considered to simplify the mathematical analysis.

\section{Mathematical Modeling}

The motion of a single electron moving in one dimension is represented by time-independent Schrödinger's equation

$$
-\frac{\hbar^{2}}{2 m^{*}} \frac{d^{2} \psi(z)}{d z^{2}}+V(z) \psi(z)=E_{z} \psi(z)
$$

For the double barrier structure under consideration as shown in Fig. 1, we consider the solutions to Schrödinger's equation within each region for electron energy less than barrier potential -

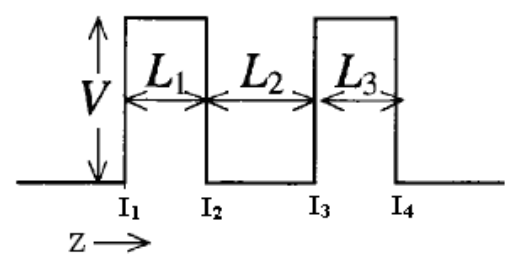

Figure 1: Schematic Structure for DBRT

$\psi(z)=A \exp \left[i \kappa_{1} z\right]+B \exp \left[-i \kappa_{1} z\right]$

for $\mathrm{z}<\mathrm{I}_{1}$

$$
\psi(z)=C \exp \left[\kappa_{2} z\right]+D \exp \left[-\kappa_{2} z\right]
$$

for $\mathrm{I}_{1}<\mathrm{Z}<\mathrm{I}_{2}$

$\psi(z)=F \exp \left[i \kappa_{1} z\right]+G \exp \left[-i \kappa_{1} z\right]$ for $\mathrm{I}_{2}<\mathrm{Z}<\mathrm{I}_{3}$

$\psi(z)=H \exp \left[\kappa_{2} z\right]+J \exp \left[-\kappa_{2} z\right]$

for $\mathrm{I}_{3}<\mathrm{z}<\mathrm{I}_{4}$

$\psi(z)=K \exp \left[i \kappa_{1} z\right]+L \exp \left[-i \kappa_{1} z\right]$

for $\mathrm{I}_{4}<\mathrm{z}$

where $\kappa_{1} \& \kappa_{2}$ are defined as:

$\kappa_{1}=\frac{\sqrt{2 m_{w}^{*} E}}{\hbar}$

$\&$

$\kappa_{2}=\frac{\sqrt{2 m_{b}^{*}(V-E)}}{\hbar}$

The positions of interfaces have been labeled $\mathrm{I}_{1}, 1_{2}, \mathrm{I}_{3}$ and $\mathrm{I}_{4}$ respectively. Using standard BenDaniel Duke boundary conditions at each interface, and introducing transfer matrix technique,

$$
\left(\begin{array}{l}
A \\
B
\end{array}\right)=M_{1}^{-1} M_{2} M_{3}^{-1} M_{4} M_{5}^{-1} M_{6} M_{7}^{-1} M_{8}\left(\begin{array}{l}
K \\
L
\end{array}\right)
$$

We assume that there are no further heterojunctions to the right of the structure, so that no further reflections can occur and wave function beyond the structure can only have a traveling wave component moving to the right, i.e. the coefficient $L$ must be zero. Thus equation (4) can be modified as:

$$
\left(\begin{array}{l}
A \\
B
\end{array}\right)=M\left(\begin{array}{l}
K \\
0
\end{array}\right)
$$

So transmission coefficient can be given by-

$T(E)=\frac{K K^{*}}{A A^{*}}=\frac{1}{M_{11}{ }^{*} M_{11}}$

For calculation of tunneling current through the complex multi barrier hetero structure, Fermi distributions are considered on both the sides which provide the probability that an electron state of certain energy is occupied. Assuming Fermi distributions on the left and right hand sides as $f_{L}$ and $f_{R}$ respectively, tunneling current is proportional to

$$
I \propto \int_{0}^{\infty} T(E)\left[f_{L}(E)-f_{R}(E)\right] D(E) d E
$$

where $D(E)$ is the density of states function, and $T(E)$ is the probability of tunneling for an electron of energy $E$. Fermi functions are given by

$$
f_{L}(E)=\frac{1}{1+\exp \left[\left(E+q V-E_{F}\right) / k_{B} T\right]}
$$

and

$$
f_{R}(E)=\frac{1}{1+\exp \left[\left(E-E_{F}\right) / k_{B} T\right]}
$$


where $E_{F}$ is the Fermi energy under zero applied field. Electrons in the left hand side of the strucutre considered have an energy which differs from the zero field by an amount $-q V$.

Density of states for a free electron Fermi gas is given as-

$$
D(E)=\frac{\sqrt{2} m_{e}^{3 / 2}}{\pi^{2} \hbar^{3}} \sqrt{E-E_{C}} \Theta\left(E-E_{C}\right)
$$

where $E_{C}$ is the energy at the bottom of the conduction band, and $\Theta\left(E-E_{C}\right)$ is the Heavyside step function.

E- $\kappa$ dispersion relationship for barrier material consists the nonparabolicity factor-

$$
E(\kappa)=\frac{\hbar^{2}}{2 m^{*}} \kappa^{2}\left(1-\gamma \kappa^{2}\right)
$$

where $\gamma$ is the coefficient of conduction band nonparabolicity, and is a function of material composition and spin-orbit splitting factor, may be written as-

$$
\gamma=\frac{\hbar^{2}}{2 m^{*}}\left(1-\frac{m^{*}}{m_{0}}\right)^{2}\left(\frac{3+4 y+2 y^{2}}{3+5 y+2 y^{2}}\right)\left(\frac{1}{E_{g}}\right)
$$

Here we consider only first higher-order term of the series. The wave functions are subjected to the variable effective mass condition at different materials where they are constant throughout the defined regions.

\section{RESULTS \& DISCUSSION}

Analysis starts with a simple idealistic double barrier structure where GaAs is surrounded by $\mathrm{Al}_{\mathrm{x}} \mathrm{Ga}_{1-\mathrm{x}} \mathrm{As}$ with equal mole fraction of $\mathrm{Al}$ on both the sides; i.e., the device can safely be termed as potentially symmetric. It is also considered that barrier potential is solely a function of the material parameters, and effective masses of barrier region and in well region have a mismatch as it depends on mole fraction of $\mathrm{Al}$ in barrier regions.

Fig. 2 gives the profile of transmission coefficient as function of energy for different well width, when barrier widths of the structure are considered equal and kept constant for the simulation purpose. For that structure, with increasing well width, tunneling probabilities can be estimated at lower energy values. But by increasing barrier width, tunneling probability decreases, as evident from Fig. 3.

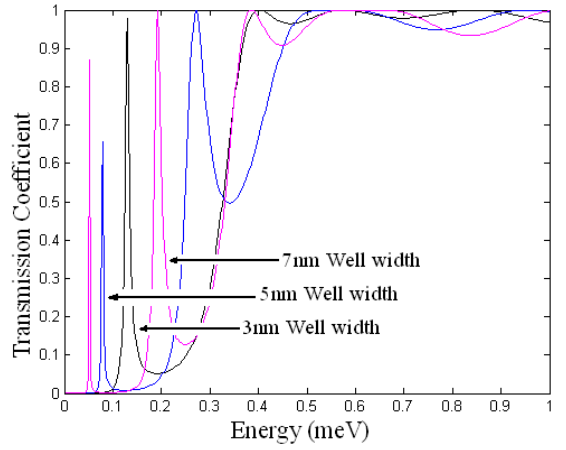

Figure 2: Transmission Coefficient Profile with Energy for Different Well Width for Parabolic Band Structure Considering GaAs/ $/ \mathrm{Al}_{0.3} \mathrm{Ga}_{0.7} \mathrm{As}$ Composition
It is quite evident from the figures that the curves contain Dirac $\delta$-functions at certain energies below the potential barrier height, which is absent in single finite quantum barrier profile. The system behaves as transparent at these resonance values. Localized wave functions of these states are nomenclature as quasi-bound states, which are not stationary.

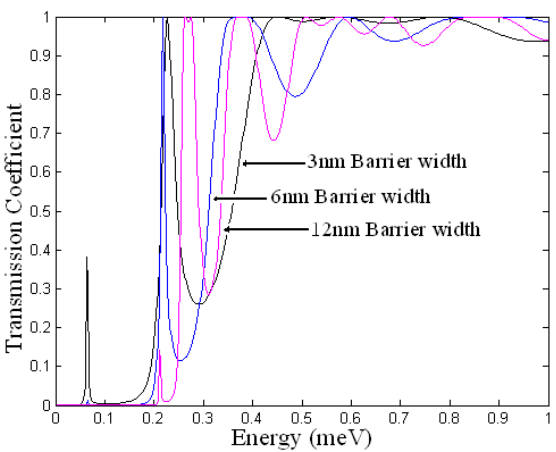

Figure 3: Transmission Coefficient Profile with Energy for Different Barrier Width for Parabolic Band Structure Considering $\mathrm{GaAs} / \mathrm{Al}_{0.3} \mathrm{Ga}_{0.7} \mathrm{As}$ Composition

With incorporation of band non parabolicity effect, it is observed that eigen state shifts towards higher energy value compared to the value obtained for parabolic band consideration. Simulation is carried out for specified material composition of the higher band gap material. It is observed that in Fig 4, incorporating band non parabolicity shifts energy state further for thicker barrier dimension. Reverse phenomenon is observed when well width is decreased, as observed from Fig 5. Smaller the width of the well, higher the shift of eigen state as band non parabolicity factor is considered. By increasing the potential barrier height through increment of $\mathrm{Al}$ mole composition, transmission probability reduces at lower energy values. This is plotted in Fig 6.

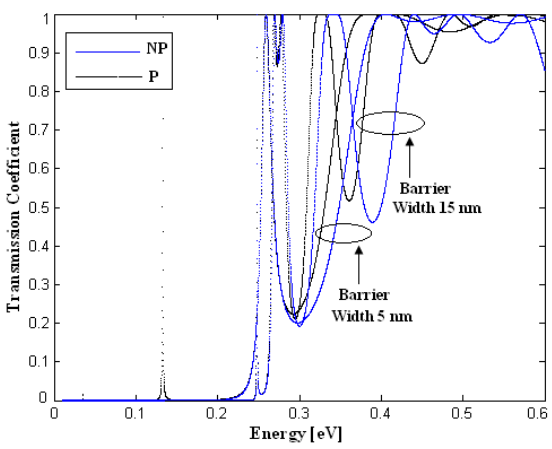

Figure 4: Comparative Analysis of Transmission Coefficient with Energy for Different Barrier width for both Parabolic and Nonparabolic Band Structure considering GaAs/ $/ \mathrm{Al}_{0.3} \mathrm{Ga}_{0.7} \mathrm{As}$ Composition

Current is computed as a function of applied voltage for different dimensional variation of the structure. From the simulation, it is observed that current peak is obtained at a lower voltage depending on the dimension of the structure. This is where resonant tunneling takes place. Just as the first peak passes, a negative differential resistance region is occurred. This region shows a downward fall of current, signifies the fact that Fermi level crosses the energy of one of the quasi-bound states in the well. 


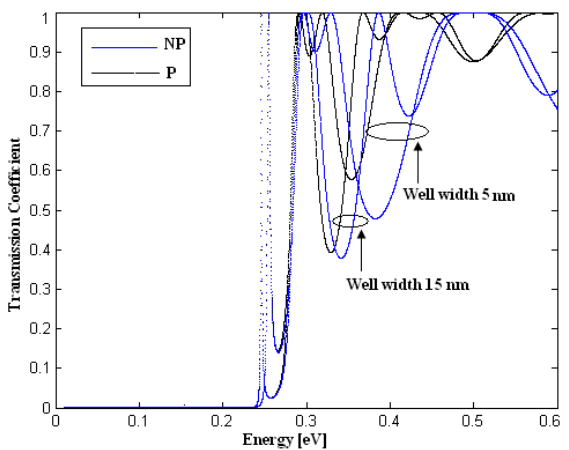

Figure 5: Comparative Analysis of Transmission Coefficient with Energy for Different Well Width for both Parabolic and Nonparabolic Band Structure considering GaAs/ $\mathrm{Al}_{0.3} \mathrm{Ga}_{0.7} \mathrm{As}$ Composition

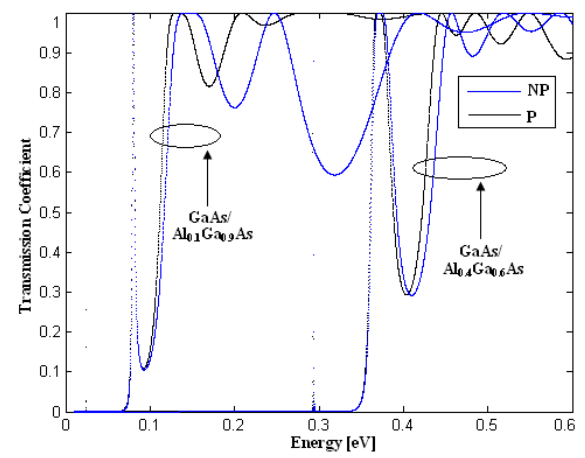

Figure 6: Comparative Analysis of Transmission Coefficient with Energy for Different Material Composition for Both Parabolic and Nonparabolic Band Structure with Constant Well and Barrier Widths

Fig 7 shows the variation of current for the double quantum barrier structure for different well width. Since with increase of well width, confinement decreases; so the first peak of the current appears at lower bias values. From the plot, it is obtained that for $5 \mathrm{~nm}$ well width; first peak appears at 0.135 Volt, whereas the same is available for $10 \mathrm{~nm}$ and $15 \mathrm{~nm}$ widths at 0.054 Volt and 0.024 Volt respectively. Also, by increasing well width, more number of peaks appears which is due to the reduction of spacing between subsequent eigenenergies. Another factor may be noted that the origin of NDR and subsequently existence of sharp valley is more prominent for lower well width. For $5 \mathrm{~nm}$ well width, difference between peak voltage and valley voltage is 0.051 Volt, whereas for $10 \mathrm{~nm}$ width, it reduces to 0.036 Volt; and for $15 \mathrm{~nm}$ width, it further reduces to 0.027 Volt.

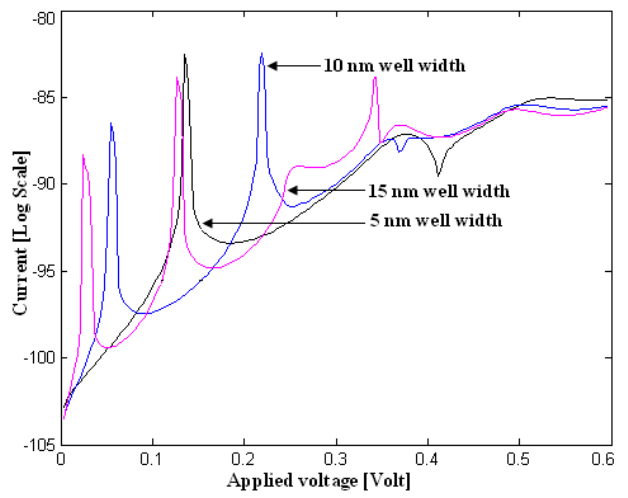

Figure 7: Current Profile with Applied Bias for Different Well

Width for Parabolic Band Structure Considering $\mathrm{GaAs} / \mathrm{Al}_{0.3} \mathrm{Ga}_{0.7} \mathrm{As}$ Composition

By varying barrier width, significant change is not expected regarding the position of peak current, but two significant points are noted from the characteristics, as shown in Fig 5. The position of second peak deviates slightly towards lower bias as the barrier with increases. From the figure, it is obtained that for 5, 10 and $15 \mathrm{~nm}$ barrier widths; second peak appears as 0.216 Volt, 0.219 Volt and 0.22 Volt respectively. Also the first valley becomes sharper as we increase the barrier width, as may be noted from the plot. Magnitude of current also decreases with increase of barrier width, as expected.

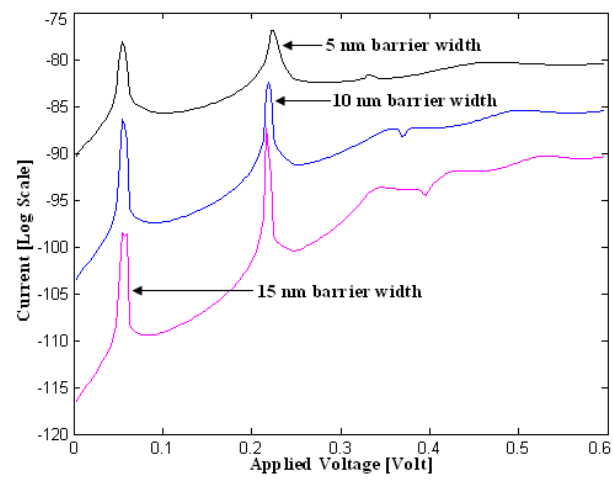

Figure 8: Current Profile with Applied Bias for Different Barrier Width for Parabolic Band Structure considering GaAs/ $/ \mathrm{Al}_{0.3} \mathrm{Ga}_{0.7}$ As Composition

For constant well and barrier dimensions, if material composition is varied, then energy-peak shifts towards higher bias value. This is plotted in Fig 9. With higher Al mole percentage, potential barrier increases, which increases quantum confinement, and thus transmission probability reduces; as is evident from Fig 6. 


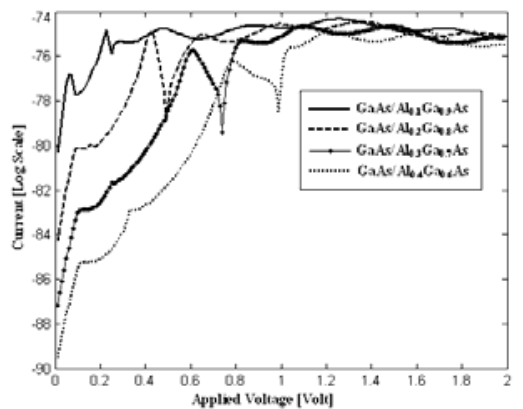

Figure 9: Current Profile with Applied Bias for Different Material Composition for Parabolic Band Structure considering Constant Well and Barrier Widths

\section{CONCLUSION}

Transmission coefficient, eigen energy and tunneling current for potentially symmetric quantum double barrier structure, and existence of quasi-bound state is verified through the occurrence of peaks in the characteristic curves. Due to the finite width and energy of potential barrier on either side of the potential well, there is a finite probability that a particle can tunnel out the potential well. Thus, we may conclude that the probability of electron tunneling through the proposed structure is finite and measurable. Also the tunneling phenomenon may be characterized by transmission and reflection coefficients, which are associated with the applied flux (equivalent to the energy given to the electrons) and material composition. Obviously it is assumed that the electrons will tunnel through one end, and won't from the other. Peaks of the transmission coefficient profiles show the resonance values. First peak energy if is lower than potential barrier energy, then it should be quite narrow. This suggests that for the particle with energy $E_{0}$ localized by the two potential barriers.

Band nonparabolicity is a critical parameter considering the precision of theoretical result, and hence should be considered when simulation is performed as it deals with more accurate dispersion relation including spin-orbit splitting factor. Comparative result shows that inclusion of Nonparabolicity-factor makes a shift of origin of eigenstates towards higher energy value. Al composition is varied within type-I heterostructure limitation so that structure can be made potentially different.

Current profiles support the existence of quasi-bound states. This, in-turn, speaks in favor of existence of NDR following each peak. Number of peaks is critically dependent on well dimension, as evident from Fig 7, but is totally independent on barrier width, can be verified from Fig 8. Also the sharpness of crossover region between Fermi level and the energy level of quasi-bound state determines the extent of valley region, which ultimately decides the PVR. Finally, it can be stated that incorporation of other complex effects such as many-electron interaction, interface scattering etc. should be considered in constructing a complete picture of resonant tunneling in double barrier resonant structures. This analysis can be further extended to analyze complex one-dimensional confined structures having arbitrary potential profiles.

\section{REFERENCES}

[1] A.R. Sugg \& J.P.C. Leburton, "Modeling of Modulation-Doped Multiple-Quantum-Well Structures in Applied Electric Fields using The Transfer-Matrix Technique", IEEE Journal of Quantum Electronics, Vol. 27, Pp. 224-231, 1991

[2] C. Pigorsch, W. Wegscheider, W. Klix and R. Stenzel, "3D-Simulation of Novel Quantum Wire Transistor", Physica Status Solidi (b), Vol. 204, Pp. 346-349, 1997.

[3] M. Kunz et. al., "High-Speed Quantum Dot Lasers and Amplifiers for Optical Data Communication", Applied Physics A., Vol. 80, Pp. 11791182, 2005.

[4] G.B. Morrison and D.T. Cassidy, "A Probability-Amplitude TransferMatrix Method for Calculating the Distribution of Light in Semiconductor Lasers", IEEE Journal of Quantum Electronics, Vol. 39, Pp. 431-437, 2003.

[5] Y. Guoa, B.L. Gu, J.Z. Yu, Z. Zeng \& Y. Kawazoe, "Resonant Tunneling in Step-Barrier Structures under an Applied Electric Field", Journal of Applied Physics, 84, Pp. 918-924, 1988.

[6] S. Vatannia \& G. Gildenblat, "Airy's Function Implementation of the Transfer-Matrix Method for Resonant Tunneling in Variably Spaced Finite Superlattices", IEEE Journal of Quantum Electronics, Vol. 32, Pp. 1093-1105, 1996

[7] L. Esaki and R. Tsu, "Superlattice and Negative differential Conductivity in Semiconductors", IBM Journal Research Division, Vol. 14, Pp. 61-65, 1970.

[8] L. Esaki \& L.L. Chang, "New Transport Phenomenon in Semiconductor Superlattice", Physical Review Letters, Vol. 33, Pp. 8-, 1974.

[9] L.A. Chanda and L.F. Eastman, "Quantum Mechanical Reflection at Triangular Planar-Doped" Potential Barriers for Transistors", Journal of Applied Physics, Vol. 53, Pp. 9165-9169, 1982.

[10] D.N. Christodoulides, A.G. Andreou, R.I. Joseph and C.R. Westgate, "Analytical Calculation of the Quantum-Mechanical Transmission Coefficient for a Triangular, Planar-Doped Ppotential Barrier", Solid State Electronics, Vol. 28, Pp. 821-822, 1985.

[11] L. Scandella and H.J. Güntherodt, "Field Emission Resonances Studied with $\mathrm{d} I / \mathrm{d} s(V)$ and $\mathrm{d} I / \mathrm{d} V(V)$ Curves", Ultramicroscopy, Vol. 42, Pp. 546$552,1992$.

[12] L.L. Chang, L. Esaki \& R. Tsu, "Resonant Tunneling in Semiconductor Double Barriers", Applied Physics Letters, Vol. 24, Pp. 593-595, 1974.

[13] M.A. Reed, R.J. Koestner \& M.W. Goodwin, "Resonant Tunneling Through a $\mathrm{HeTe} / \mathrm{Hg}_{1-\mathrm{x}} \mathrm{Cd}_{\mathrm{x}} \mathrm{Te}$ Double Barrier, Single Quantum Well Structure", Journal of Vacuum Science and Technology A, Vol. 5, Pp. 3147-3149, 1986.

[14] R. Wessel \& M. Alterelli, "Quasi Stationary Energy Level Calculation for Thin Double Barrier GaAs- $\mathrm{Ga}_{1-\mathrm{x}} \mathrm{Al}_{\mathrm{x}} \mathrm{As}$ Heterostructures", Physical Review B, Vol. 39, Pp. 10246-10250, 1989.

[15] D.J. BenDaniel and C.B. Duke, "Space-Charge effects on Electron Tunneling", Physical review, Vol. 152, Pp. 683-692, 1966.

[16] R.C. Miller, D.A. Kleinman and A.C. Gossard, "Energy-Gap Discontinuities and Effective Masses for GaAs- $\mathrm{Al}_{x} \mathrm{Ga}_{1-\mathrm{x}} \mathrm{As}$ Quantum Wells", Physical Review B, Vol. 29, Pp. 7085-7087, 1984.

[17] T. Hiroshima and R. Lang, "Effect of Conduction Band Nonparabolicity on Quantized Energy Levels of a Quantum Well", Applied Physics Letters, Vol. 49, Pp. 456-457, 1986.

[18] D.P. Nelson, R.C. Miller and D.A. Kleinman, "Band Nonparabolicity Effects in Semiconductor Quantum Wells", Physical Review B, Vol. 35, Pp. 7770-7773, 1987.

[19] D.P. Dave, "Numerical Technique to Calculate Eigenenergies and Eigenstates of Quantum Wells with Arbitrary Potential Profiles", IET Electronics Letters, Vol. 27, Pp. 1735-1737, 1991.

[20] E. Anemogiannis, "Bound and Quasibound State Calculations for Biased/Unbiased Semiconductor Quantum Heterostructures", IEEE Journal of Quantum Electronics, Vol. 29, Pp. 2731-2740, 1993.

[21] G. Bastard, E.E. Mendez, L.L. Chang \& L. Esaki, "Variational Calculations on a Quantum Well in an Electric Field", Physical Review B, Vol. 28, Pp. 3241-3245, 1983.

[22] A.K. Ghatak, K. Thyagarajan \& M.R. Shenoy, "A Novel Numerical Technique for Solving the One-Dimensional Schrödinger Equation using Matrix Approach - Application to Quantum Well Structures", IEEE Journal of Quantum Electronics, Vol. 24, Pp. 1524-1531, 1988.

[23] K.F. Brennan and C.J. Summers, "Theory of Resonant Tunneling in a Variably Spaced Multiquantum Well Structure: An Airy Function Approach", Journal of Applied Physics, Vol. 61, Pp. 614-623, 1987. 
[24] K. Hayata, M. Koshiba, K. Nakamura \& A. Shimizu, "Eigenstate Calculations of Quantum Well Structures using Finite Elements", Electronics Letters, Vol. 24, Pp. 614-616, 1988.

[25] E.P. Samuel \& D.S. Patil, "Analysis of Wavefunction Distribution in Quantum Well Biased Laser Diode using Transfer Matrix Method", Progress In Electromagnetics Research Letters, Vol. 1, Pp. 119-128, 2008.

[26] K. Talele \& D.S. Patil, "Analysis of Wavefunction, Energy and Transmission Coefficients in GaN/AlGaN Superlattice Nanostructures", Progress In Electromagnetics Research Letters, Vol. 81, Pp. 237-252, 2008.

[27] B. Jonsson \& S.T. Eng, "Solving the Schrödinger Equation in Arbitary Quantum-Well Profiles using the Transfer-Matrix Method", IEEE Journal of Quantum Electronics, Vol. 26, Pp. 2025-2035, 1990.

[28] Y. Tsuji and M. Koshiba, "Analysis of Complex Eigenenergies of an Electron in Two- and Three-Dimensionally Confined Systems using the Weighted Potential Method", Microelectronics Journal, Vol. 30, Pp. 1001-1006, 1999.

[29] P.H. Rivera and P.A. Schulz, "Analysis of the Current-Voltage Characteristics Lineshapes of Resonant Tunneling Diodes", Brazilian journal of Physics, Vol. 26, Pp. 304-307, 1996.

[30] S.S. Allen \& S.L. Richardson, "Theoretical Investigations of Resonant Tunneling in Asymmetric Multibarrier Semiconductor Heterostructures in an Applied Constant Electric Field", Physical Review B, Vol. 50, Pp. 11693-11700, 1994. 